\title{
Avionics Architectures for Exploration: Wireless Technologies and Human Spaceflight
}

\author{
Montgomery B. Goforth, James E. Ratliff, Richard J. Barton, Raymond S. Wagner, and Chatwin Lansdowne \\ NASA Johnson Space Center (JSC) \\ Houston, TX, 77058, USA
}

\begin{abstract}
The authors describe ongoing efforts by the Avionics Architectures for Exploration (AAE) project chartered by NASA's Advanced Exploration Systems (AES) Program to evaluate new avionics architectures and technologies, provide objective comparisons of them, and mature selected technologies for flight and for use by other AES projects. The AAE project team includes members from most NASA centers and from industry. This paper provides an overview of recent AAE efforts, with particular emphasis on the wireless technologies being evaluated under AES to support human spaceflight.
\end{abstract}

Keywords-Wireless, Avionics

\section{INTRODUCTION}

The field of Avionics is advancing far more rapidly in terrestrial applications than in spaceflight applications. Spaceflight Avionics are not keeping pace with expectations set by terrestrial experience, nor are they keeping pace with the need for increasingly complex automation and crew interfaces providing crew autonomy as we move beyond Low Earth Orbit. NASA must take advantage of the strides by both spacerelated and terrestrial industries to drive our development and sustaining costs down.

It is the intent of the Avionics Architectures for Exploration (AAE) team to develop a common core avionic system that has standard capabilities and interfaces, and contains the basic elements and functionality needed for any spacecraft. This common core will be scalable and tailored to specific missions. It will incorporate hardware and software from multiple vendors, and be upgradeable in order to infuse incremental capabilities and new technologies. It will maximize the use of reconfigurable open source software (e.g., Goddard Space Flight Center's (GSFC) Core Flight Software (CFS)).

The long-term focus is on improving functionality, reliability, and autonomy, while reducing size, weight, and power. Where possible, the project will leverage terrestrial commercial capabilities to drive down development and sustaining costs. We will select promising technologies for evaluation, compare them in an objective manner, and mature them to be available for future programs. Of particular interest to the project is the use of wireless technologies, which are the primary focus of this paper.

\section{APPROACH}

The overall approach of the AAE project emphasizes the need for testing of multiple alternatives to provide objective evaluations of the relative merits of architectures and

The authors would like to acknowledge the support of the AAE sponsors at NASA Headquarters: Mr. Jason Crusan (Director, Advance Exploration Systems Division) and Mr. Richard McGinnis (Lead, AES Operations Domain). technologies. Technologies selected for evaluation include both "legacy" systems (e.g., MIL-STD-1553B), and those included in technology roadmaps produced by NASA's Office of Chief Technologist (OCT), Avionics Steering Committee (ASC), and Space Communications and Navigation (SCaN) Office. We recognize that any future exploration vehicles will likely be composed of a cluster of more specialized vehicles deployed at different times by various organizations/contractors (perhaps from different countries), and that we must address how these specialized vehicles interact during all mission phases. Although the project is focused on avionics for human spaceflight, we are considering technologies applicable for both crewed and robotic vehicles. The AAE project emphasizes the investigation of human interfaces, more powerful processors and network configurations, wireless technologies for both networking and instrumentation, and flexible longhaul communications technology.

\section{A. Challenges and Guidelines}

As part of the initial AAE effort, a set of high-level challenges and associated architectural guidelines have been identified. The challenges are as follows:

- $\quad$ Future exploration vehicles are undefined, but are likely to be an aggregate of multiple vehicles from multiple sources. This will drive sparing, redundancy, etc.

- Size, weight, and power (SWaP) must always be minimized.

- Processing requirements exceed that which can be provided by existing space hardened avionics (e.g., Power PC-based Rad750).

- $\quad$ The radiation environment at high Earth orbit (HEO) and beyond is much worse than it is at low earth orbit (LEO).

- Because of the radiation environment, we cannot rely on commercial-off-the-shelf (COTS) hardware for additional processing capabilities as NASA has done on Shuttle and the International Space Station (ISS) (i.e., laptops are not likely to work reliably).

- Exploration vehicle requirements will change/grow over the vehicle's lifetime, as will the expectations set by terrestrial state of the art. We need to accommodate these changes without undue expense.

- A distributed ground development and test architecture must be supportable. This requires loosely-coupled interfaces between systems. 
These high level challenges, along with other project level decisions, lead to the following set of architectural guidelines:

- Minimize Avionics SWaP in the Flight Vehicle. Use wireless local area networks (LANs) and sensor networks where possible. Use low-power or passive (no power) sensors whenever practical. Minimize total wiring on the vehicle. Minimize unique components for sparing.

- Keep the architecture and design modular so we can launch incrementally. Allow capabilities to be integrated into the vehicle when they are needed, or earlier if it makes sense from an available launch mass perspective.

- Minimize Cost. Use existing capabilities to avoid nearterm design, development, test, and evaluation (DDT\&E). Allow for growth using new technology to avoid future DDT\&E. Allow for infusion of new technology to reduce sustaining effort. Look for places where improved Avionics can drive down overall vehicle costs.

- Minimize Risk. Use proven technology for critical functions. Use existing capabilities to minimize schedule risk.

- Minimize logistics and maintenance. Pay particular attention to the trade-off between utilizing precious habitable volume for mounting avionics inside the spacecraft versus the effort required for external maintenance via extra-vehicular activity (EVA).

- Support Heterogeneity. We cannot expect every module of an aggregate vehicle to be the same. No one architecture/design will be an acceptable answer for everything.

- Strive for "Commonality". This can mean choosing the same components/boards/boxes to be used throughout a vehicle. It must mean developing a way for these different things to talk to each other. It should mean making sure that things of a similar type can be exchanged to allow for minimal sparing.

- Vehicle backbone network. A high-speed wired LAN (e.g., Ethernet) will be available for both crew support and as a backbone network for non-critical vehicle data transport.

- Maximize the use of Core Flight Software (CFS). Another AES-funded effort at JSC is the Core Flight Software (CFS) project. Briefly stated, the Core Flight Software project's objective is to evolve and extend the reusability of GSFC's Core Flight Software System into human-rated systems, thus enabling low cost, and rapid access to space. It was decided that the AAE project would make maximum use of CFS. This approach should maximize our ability to leverage platforms, resources and skills from synergetic programs/projects for development of next generation human-rated space software systems and utilize these products in direct support of development and certification of future manned programs.Incorporate “Loose” Interfaces. Wireless communication interfaces are by nature loosely-coupled but usually co-located because they are unwieldy. It is sometimes highly desirable to test parts of a vehicle "together" while still at the respective vendor facilities. For both reasons, internal interfaces must consider how a system can be distributed during development, integration, and checkout.

- Use iPAS and F.F. In order to maximize return on investment, the AAE project has made extensive use of the Flight Deck of the Future (F.F) and the Integrated Power, Avionics, and Software (iPAS) capability developed by JSC Engineering. iPAS is a multi-system environment where next generation flight systems can be tested and demonstrated. It provides multi-mission/multivehicle simulations, a common set of test services, access to a variety of actual sensors and effectors, and access via the Distributed Systems Network (DSNet) to capabilities at multiple NASA centers ${ }^{1}$. The F.F focuses primarily on the human-machine interface. It allows us to evaluate different interface technologies together with personnel from Flight Crew Operations, Human Health and Performance, and other stakeholders.

\section{PROGRESS TO DATE AND CURRENT STATUS}

To date, the AAE Project has successfully demonstrated a plausible avionics architecture for a notional habitat and waystation located at the L2 Earth-Moon Lagrange point. We have also made significant strides toward the goal of a flexible avionics architecture that can be used to evaluate future concepts/architectures/components for both our nominal L2 Station and other vehicles [1,2,3].

The AAE architecture emphasizes the use of open interface standards and reconfigurable open source software. It also contains the basic core elements and functionality required for any spacecraft while providing scalability and reconfigurablility for different missions. The goal is to enable integration of hardware from multiple vendors and international partners and to provide the capability to evaluate and use evolving (near launch) technology. This robust architecture will allow us to continuously upgrade our capabilities and infuse new technologies with cost effective validation.

Our efforts remain centered on incremental architectural upgrades applied to different mission scenarios. These upgrades and scenarios are evaluated during periodic Integrated Tests (IT's). To date, six IT's have been conducted. The remaining sub-sections of Section III describe the goals and accomplishments to date for each of our technical Areas of Emphasis (AOEs). Details are provided only for AOEs focused on wireless technology. The plans and strategies for the future are described in Section IV.

\section{A. Proximity Communications}

As we accept the transformation of the vehicle backbone network to Internet Protocol (IP), and vehicle communications with the ground become entirely IP packet-based, proximity communications comes to mean a wireless extension of the wired vehicle backbone network, while radios providing backhaul from the vehicle to the ground become gateways to the terrestrial networks. 
A number of widely commercialized open standards fulfill sophisticated terrestrial needs for proximity communications. These investments can be heavily leveraged to support space exploration scenarios. Miniaturized consumer-grade parts often have sufficient radiation survivability to be useful. Customarily these technologies are manually configuration-managed by the Information Technology trade and by users. Technologies of distinct interest include Wi-Fi (IEEE 802.11n,ac), Wi-Gig (802.11ad) and LTE. Overlapping these technologies could produce a composite wireless network that provides useful coverage beyond $100 \mathrm{~km}$ at a several Mbps, tapering to many Gbps for supporting live video at $100 \mathrm{~m}$.

For space exploration, the network architecture must be robust, scalable and extensible over time by a variety of vendors, adaptable to unforeseen mission objectives, require minimal pre-deployed infrastructure, require minimal crew time for maintenance, and be manageable over intermittent high-delay communication links. It is therefore important to achieve a standards-based autonomous, self-organizing, selfhealing wireless network based on the principle of cooperative communication. [4]. By including nodes with several types of communication peripherals each (Wi-Gig, Wi-Fi, LTE), a proximity network can be formed of a collection of meshes with frequent cross-links. An example of this is illustrated in Figure 1.

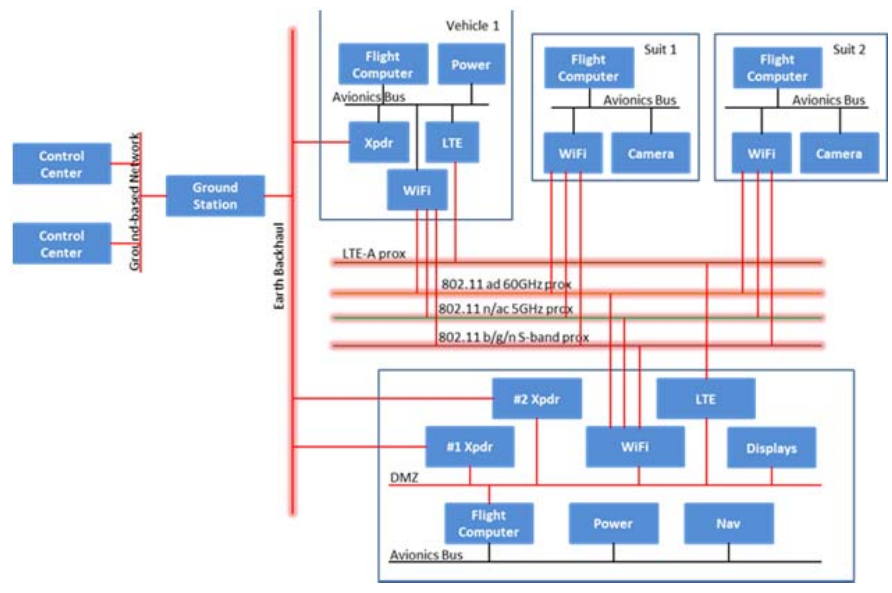

Figure 1. Notional Wireless Proximity Mesh Network.

We must then consider what behavior we want when the proximity coverage of two vehicles overlaps. Do the networks join? Does that mean the two vehicles become one? Can the crew of one see a docking camera on the other? What if the vehicles belong to partnered political allies? What can applications on one vehicle access on the other? Suppose two vehicles dock in LEO under cover of a network of geosynchronous satellites, and subsequently depart for deep space. They are both mobile and their network service provider changes, but does this mean their IP addresses both change? Questions like these can be investigated using alternative iPAS scenarios and solutions can be informally evaluated using iPAS, distributed vehicle assets, and affiliated ground station and control center assets.

\section{B. Wireless Sensing}

Wireless sensing represents a key enabling technology for next generation space vehicles. Substantial weight reductions may be realized by eliminating the cable runs, cable harnesses, and connectors common to wired instrumentation systems in modern vehicles and launchers. These weight savings may then be applied to increase the capabilities of the vehicle itself or permit more vehicle payload, expanding the mission scope. In addition, allowing sensors to be entirely free of wires, both for power and communication, affords unprecedented flexibility in sensor installation. Removing physical interfaces to vehicle power and data buses reduces the burden on system integrators, especially where bulkhead penetrations are required to route cable runs. This can allow sensor installation much later than normal in a vehicle's assembly, integration, and testing phase, and it can also allow installation of new instrumentation once a vehicle is deployed operationally, in the case of crewed systems such as the International Space Station (ISS). The ISS, which is well into its second decade of operation and was on the drawing board more than a decade prior to the launch of its first elements, provides an excellent example of a vehicle whose sensing needs late in life could not be anticipated in their entirety by its designers.

Sensing requirements on a vehicle can cover a wide range, extending from occasional sampling of environmental data (such as $\mathrm{CO}_{2}$ levels in a habitable module) for increased situational awareness to capturing high-bandwidth waveforms (such as accelerometer data) as part of a developmental flight instrumentation (DFI) task to study vehicle performance and structural health. Data may be gathered and transmitted periodically according to some set schedule or on demand in response to some event such as a micrometeoroid impact.

As with the other AAE technology areas discussed in this paper, we focus on leveraging commercial wireless data transport standards and COTS radios as much as possible, given the rapid progress made over the last decade in the related fields of wireless sensor networks (WSNs) and the Internet of Things (IoT). Though a number of alternatives are on the market, covering a wide gamut of sensing needs, we have focused on three distinct protocols thus far: ISA100.11a, IEEE 802.11 (Wi-Fi), and EPC Global Class 1 Generation 2. These three cover a range of applications from very low to very high data rates and entail different tradeoffs between performance and power consumption. Bluetooth and BLE are also finding an entry in the area of wearables, but are not treated here.

ISA100.11a-2011, from the Industrial Society of Automation, describes a reliable, low power, low data-rate, multi-hop mesh network. It starts with the $2.4 \mathrm{GHz}$ physical (PHY) layer of the IEEE 802.15.4-2006 standard and builds a complete protocol stack as described by the Open Systems Interconnection (OSI) model. A channel-hopping scheme, centrally coordinated by a network management, gives the protocol a robustness lacking in the fixed-channel IEEE 802.15.4 standard and allows the network to adaptively contend with time-varying interferers such as other wireless systems occupying same band. Thus, it is more appropriate for wireless monitoring and control in harsh industrial 
deployments, and by extension spaceflight applications, than plain IEEE 802.15.4 or the derivative ZigBee Pro protocol. [5]

ISA100.11a shares many features in common with the similar WirelessHART standard, and it also forms the basis for the IEEE 802.15.4e amendment to the 802.15.4 standard. We focus on deploying ISA100.11a due to the availability of VN210 radios from Nivis, LLC and the ease of applicationlayer integration to arbitrary applications, but the results generalize. Our choice of ISA100.11a is consistent with the Consultative Committee for Space Data Systems (CCSDS) "Magenta Book" recommendation for low data-rate wireless communications for spacecraft monitoring and control [6].

To evaluate this standard, we have interfaced the Nivis ISA100.11a radio to a power supply and Texas Instruments MSP430-family microcontroller using a modular form factor developed at JSC, similar to that depicted in Figure 2. The stack is fitted with a module allowing an interface to a thruster pressure transducer, enabling us to monitor thruster firings from a bank of cold-gas, computer-controlled jets in the iPAS testbed.

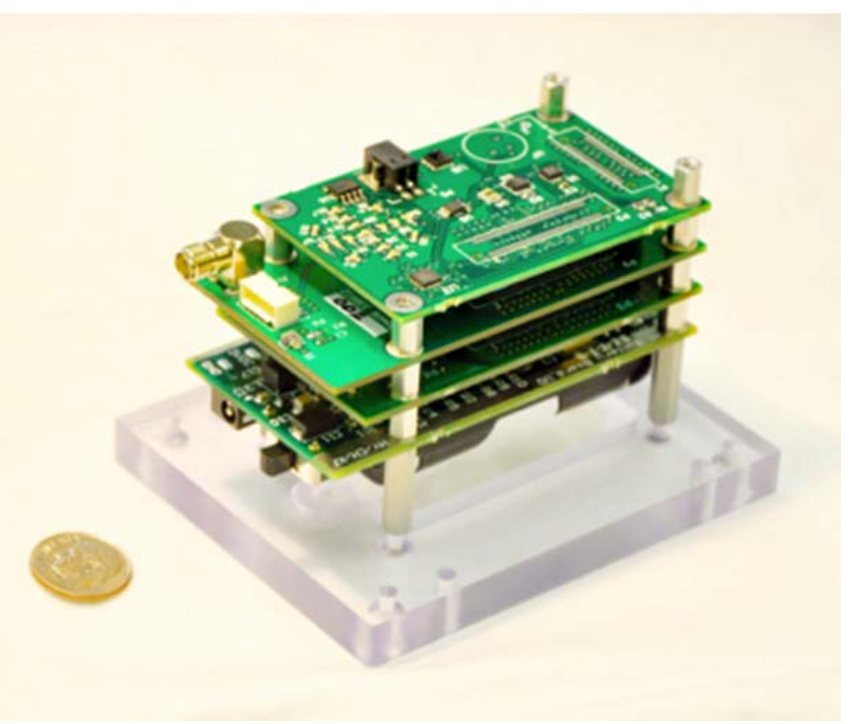

Figure 2: ISA100.11a modular wireless sensor node.

Although the wireless thruster sensor itself captures fullbandwidth pressure waveforms, thruster firing events are summarized before being transmitted over the low data-rate ISA100.11a network. When a pressure transient is detected (a set threshold is exceeded), an "on" message is sent, followed by an "off" message when the transient ends (by a second threshold crossing) that contains a summary of the transient event consisting of times and pressure readings for the starting/ending threshold crossings and the transient peak. Sensor nodes communicate with the ISA100.11a system manager and network gateway, co-located in the iPAS testbed, and data is passed to displays in the F.F via a CFS application.

This ISA100.11a application demonstrates use of a low data-rate, low-power "active” WSN - so dubbed because the radio requires active application of power to communicate. To explore high-data rate active wireless sensing, we repeat the exercise described above, substituting an IEEE 802.11n (Wi-
Fi) compliant, 2.4 GHz COTS radio module from Gainspan for the Nivis ISA100.11a radio. In this case, the entire thruster pressure waveform is transmitted, rather than a simple 3element ordered pair summary, taking advantage of the much greater throughput afforded by IEEE 802.11.

Finally, we round out our AAE investigation of wireless sensing by focusing on so-called "passive" transmission schemes. In contrast to the active schemes like ISA100.11a and IEEE 802.11n, these passive schemes require no application of onboard power to drive the transmitters; rather, they make user of power beamed at them by an interrogator to which they then send their data. We turn to the EPC Global, Class 1 Generation 2 Radio Frequency Identification (RFID) protocol, which is typically used to track tagged items by their identifier but which also has provisions for storing sensor data as well.

Using a modular approach similar to that described for ISA100.11a and Wi-Fi, we have assembled a small test unit to interface a thermocouple to a serial-writable RFID chip from Cypress Semiconductor. Data is sampled by the RFID sensor node at a continuous rate and stored to tag memory. Rather than requiring immediate transfer of that data to the RFID interrogator, we assume the interrogator is a mobile unit which may not always be available when a new sample is taken. A network overlay inspired by delay tolerant network (DTN) techniques manages movement of sensor data between the sensor tag and the mobile interrogator; design of that overlay is described in detail in the companion paper found in [7]. Data is gathered by the mobile interrogator shown in Figure 3 and sent to a standalone console in iPAS for display.

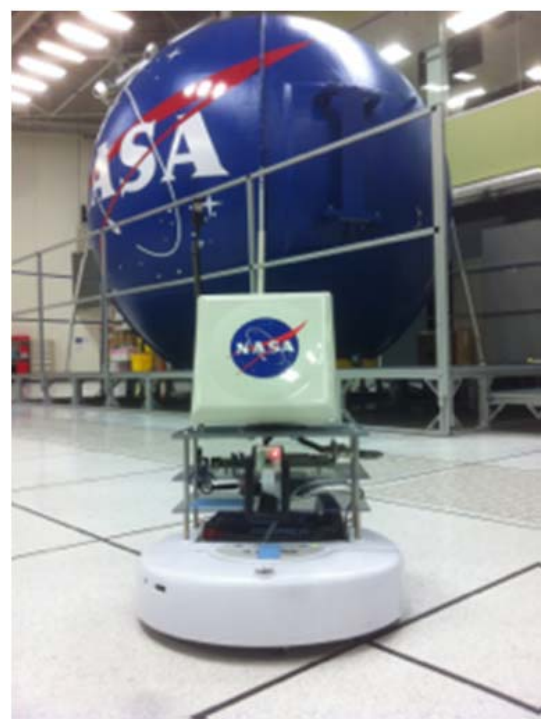

Figure 3: mobile RFID interrogator platform.

\section{Processors, Networks, and Instrumentation (PNI)}

We have successfully loaded CFS on multiple single board computers with different operating systems, and we continue to add to the combinations we have demonstrated. For the primary flight computer in the architecture we have developed a method of hot backup. This was demonstrated by the ability to simultaneously operate two dissimilar machines running dissimilar operating systems over an Ethernet network. This capability has been extended to provide a standalone CFS quad 
voting software demonstration that utilizes three unique combinations of processor and operating system. In the near future, this standalone capability will be merged with the integrated AAE architecture.

\section{Human Interfaces}

We have provided a number of system-level displays for use in the Orion Multi-purpose Crew Vehicle (MPCV) cockpit mock-up, and we are now exploring alternative display development system techniques for rapid prototyping and testing. We have successfully demonstrated our ability to render displays by implementing a radiation tolerant software GPU on an AiTech C903, and we are in the process of porting it to an AiTech C925 to take advantage of the PCIe bus on this board. We are collaborating with Kennedy Space Center (KSC) and the University of Florida-led Center for HighPerformance Reconfigurable Computing (CHREC) to identify processor architectural options for future consideration.

\section{E. Model-Based System Engineering}

NASA has initiated efforts to implement Model-Based System Engineering (MBSE). In an effort to gain experience in this area, the AAE project adopted MBSE for our reference implementations. In the near-term, MBSE tools provide with the flexibility to analyze and document a variety of architectures and share this information with other AES projects. Ultimately, this will support the AAE goal of developing a reference implementation of the avionics architecture(s) that can be provided to industry (users) as a basis for standards and procurements.

\section{F. Software Defined Radio and Delay Tolerant Networks}

To support the AAE long range communications architecture, we have been exploring the use of softwaredefined radio (SDR) technology, in which the radio transceiver performs the baseband processing functions entirely in software, including modulation/demodulation, error correction coding, and compression/decompression. SDR technologies will be of great benefit to NASA as they allow for increased interoperability, interference mitigation, higher spectral efficiency, and lower upgrade costs than application-specific hardware.

DTN is also a fundamental part of our communications architecture. NASA is developing the DTN protocol suite that extends terrestrial internet capabilities into highly stressed data communication environments where conventional internet protocols do not work well. The DTN protocol suite is being standardized by the Consultative Committee for Space Data Systems (CCSDS) and all of the DTN protocols will be international standards, supported by open-source software that can help users implement new capabilities.

The development of the DTN protocol suite is not within the scope of the AAE project, but we are incorporating new DTN capabilities as they are developed. More importantly, we are performing trade studies to determine the best location to host DTN within the spacecraft architecture, including key considerations such as size, weight and power, processor utilization, storage capacity, device real estate, and required data rates. The AAE project is also studying the impact of having DTN nodes at other locations within the end-to-end communication architecture, considering factors such as network reliability, implementation costs, and the need for international interoperability.

\section{Future StRATEGY AND GOALS}

In fiscal year 2015 (FY15), the AAE Project will be combined with the Core Flight Software (CFS) Project, the DTN Project, and Fault Detection Isolation and Recovery (FDIR) and Planning aspects of the Autonomous Mission Operations (AMO) Project. This new AES project will be known as the AES Avionics \& Software Project. The purpose of the Avionics \& Software project is to build a suite of tested and reusable avionics and software components that reduce cost and risk for future exploration programs and enable infusion of new technologies and capabilities into current Programs. This project will utilize Model-Based Systems Engineering tools to catalog the suite of avionics and software components, enabling trade-study analyses and overall system design to be easily performed based on mission requirements.

The top level goals for this project are to: 1) develop avionics and software architectures that support NASA goals for Beyond-LEO exploration "space vehicles" that may be modular, multi-vendor, and multi-IP configurations, and 2) evaluate design concepts in a system environment to verify ability to integrate diverse interfaces and evaluate system performance. We will be working towards an "open" architecture that allows use of hardware from multiple vendors, enables use of evolved technology, and provides the ability to upgrade capabilities and infuse new technologies in a cost effective manner.

With respect to wireless technology development and infusion, we will continue to study proximity communication architectures based on heterogeneous multi-hop mesh networks The most promising approaches will be infused into the iPAS testbed and evaluated under multiple scenarios in order to determine their capabilities for supporting operations in the vicinity of future exploration vehicles and habitats. We will continue to evaluate both active and passive wireless sensor networking technology and work toward the integration and testing of independent wireless subsystems with CFS and with vehicle command and data handling systems. We will also continue developmental work on the DTN protocol suite and study such aspects as DTN network administration, security, and routing within the AAE framework.

Although specific plans and objectives are still in-work, we intend to evaluate additional architectures providing redundancy with dissimilar hardware, fault management and advanced caution and warning. We will also be simulating more dynamic situations such as entry, descent, and landing. We will be supporting other AES projects such as Advanced ECLSS and EAM. We expect that some of our technology demonstrations will prove useful to both ISS and Orion.

\section{CONCLUSIONS}

During its initial phases, the AAE Project has successfully demonstrate a plausible avionics architecture for a notional L2 Station and made significant strides toward our goal of a flexible avionics architecture that can be used to evaluate future concepts/architectures/components for both a nominal 
L2 Station and other vehicles. Our Rev. 2.0 architecture provided a common core system that has standard capabilities and interfaces, and contains basic core elements and functionality needed for any spacecraft. If desired, this system could be scaled and tailored to any specified mission. The system incorporates hardware from multiple vendors, and reusable and reconfigurable open source software (e.g., GSFC CFS).

- The vision for the consolidated FY15 Avionics \& Software Project includes the development of architectures and system designs which can be used for flight programs in both the near and long term.

For the near term, this means that we must provide a point solution targeted for an identified mission in 2-3 years. Essentially this solution must be a "good enough" answer from the options we have, which can be matured for flight with minimal effort and used as a basis for procurement specifications.

For the long term, our intent is to build a "catalog" of multiple solutions which can be used "mostly off-the-shelf" for a variety of situations. This catalog will include specific components and overall architectures. Recognizing that one size does not fit all, each will be rated for suitability to different mission types. New technologies will be incorporated in a timely manner in order to take advantage of strides being made by industry to drive program development and sustaining costs down. This strategy should allow us to include vehicle "hooks and scars" for later augmentation; facilitate component repair, replacement, and upgrades; and take advantage of schedule slips during the development phase with improved hardware.
We remain committed to demonstrating the technical and economic benefits of our approach, but the amount of progress we are able to make is dependent on funding and resource constraints, along with the priorities set by the Agency for Human Spaceflight.

Our team already includes participants from most NASA centers and industry, but we recognize the need to widen participation from NASA and other government agencies, add more industry and academic partners, and begin discussions with potential international partners during the coming years. We look forward to engaging these future stakeholders.

\section{REFERENCES}

[1] W. Othon, "iPAS: AES Flight System Technology Maturation for Human Spaceflight”, AIAA Space Ops 2014 (AIAA 2014-1925).

[2] M. Goforth, J. Ratliff, K, Hames, S. Vitalpur, "Avionics Architectures for Exploration: Building a Better Approach for (Human) Spaceflight Avionics”, AIAA Space Ops 2014 (AIAA 2014-1604).

[3] M. Goforth, J. Ratliff, K, Hames, S. Vitalpur, K. Woodman, "Avionics Architectures for Exploration: Ongoing Efforts in Human Spaceflight", AIAA SPACE 2014 (AIAA 2014-4315).

[4] M. DeCristofaro et al., "Heterogeneous Wireless Mesh Network Technology Evaluation for Space Proximity and Surface Applications,” DOI: 10.2514/6.2014-1600, AIAA SpaceOps 2014 Conference, May 2014.

[5] R. Wagner and R. Barton, "Performance Comparison of Wireless Sensor Network Standard Protocols in an Aerospace Environment: ISA100.11a and ZigBee Pro”, Proc. IEEE Aerospace Conference, March, 2012.

[6] Spacecraft Onboard Interface Systems--Low Data-Rate Wireless Communications for Spacecraft Monitoring and Control. Consultative Committee for Space Data Systems (CCSDS) Magenta Book. Issue 1. May 2013.

[7] R. Wagner and R. Barton, "Delay Tolerant, Radio Frequency Identification (RFID)-enabled Sensing", Proc. IEEE Intl. Conf. on Wireless for Space and Extreme Environments (WiSEE), October, 2014. 